CERN-TH/96-72

$\mathrm{BI}-\mathrm{TP} / 96-43$

\title{
ON THE SUM RULE APPROACH TO QUARKONIUM-HADRON INTERACTIONS
}

\author{
D. Kharzeev ${ }^{1}$, H. Satz ${ }^{1,2}$, A. Syamtomov ${ }^{3}$ and G. Zinovjev ${ }^{3}$ \\ 1 Fakultät für Physik, Universität Bielefeld, D-33501 Bielefeld, Germany \\ 2 Theory Division, CERN, CH-1211 Geneva, Switzerland \\ 3 Bogolyubov Institute for Theoretical Physics, 252143 Kiev-143, Ukraine
}

\begin{abstract}
We extend a recent sum rule calculation for inelastic quarkonium-hadron interactions to realistic parton distribution functions; we also include finite target-mass corrections. Both modifications are shown to have no significant effect on the resulting cross section behaviour but the performed analysis gives useful insights on the sum-rule approach in general.
\end{abstract}

CERN-TH/96-72

BI-TP/96-43

May 1996 
In a recent study [1], the cross section $\sigma_{N \Phi}(s)$ for inelastic quarkonium-nucleon collisions was calculated from sum rules established on the basis of the operator-product expansion [2]- [6]; here $\Phi$ denotes a quarkonium ground state $(\mathrm{J} / \psi$ or $\Upsilon)$ and $s$ the squared center of mass collision energy. In order to simplify the calculations a number of approximations was made in this investigation. The aim of the present work is to obtain $\sigma_{h \Phi}(s)$ avoiding such approximations and to clarify some aspects of the sum rule approach in general.

In the formalism developed to describe the interaction of hadrons with heavy $q \bar{q}$ mesons [2], [7], 8], the large quark mass $m_{Q}$ allows one to write down the corresponding Born amplitude in the form of operator product expansion (OPE). The effects governed by short-distance physics, including the structure of heavy quarkonium state, factorize into coefficient functions, $C_{n}$, while the thorough dependence on soft structure of hadron is determined by low-energy matrix elements of gauge-field local operators, $\mathcal{O}_{n}$, renormalized at quarkonium mass scale $M_{\Phi}$,

$$
\mathcal{M}_{\text {Born }}=\sum_{n=2,4, \ldots}^{\infty} C_{n}\left\langle\mathcal{O}_{n}\right\rangle
$$

The operators entering (1) are ordered by their dimensions. If the characteristic distances of internal $q \bar{q}$ dynamics are small enough as compared to scale of QCD-vacuum fluctuations inside the hadron, only the terms of lowest dimension contribute. In the framework of the multipole expansion formalism [7], [8], the lowest dimension terms correspond to the dipole field contribution. For the sake of simplicity, we have dropped in (1) spin indices which otherwise mark the amplitudes describing the forward scattering of heavy quarkonia with different polarizations. If we restrict ourselves to the case of spin-averaged interactions, then Lorentz indices may appear only in the following combination:

$$
\mathcal{O}_{n}=\frac{1}{M_{\Phi}^{n}} K_{\mu_{1}} \ldots K_{\mu_{n}} \theta_{\mu_{1} \ldots \mu_{n}}^{G}
$$

where

$$
\theta_{\mu_{1} \ldots \mu_{n}}^{G}=i^{n-2}\left[G_{\mu_{1} \nu} \mathcal{D}_{\mu_{2}} \ldots \mathcal{D}_{\mu_{n-1}} G_{\mu_{n} \nu}\right](\text { symmetrized })-\text { traces } .
$$

Here $K_{\mu}$ is the quarkonium momentum, $G$ is the gluon field operator and $\mathcal{D}$ means the covariant derivative.

The target matrix elements of these operators are completely specified by phenomenological parameters $A_{n}$ of dimension defined as twist and the spin-averaged tensor structure,

$$
\left\langle p\left|\theta_{\mu_{1} \ldots \mu_{n}}^{G}\right| p\right\rangle=A_{n}\left(p^{\mu_{1}} \ldots p^{\mu_{n}}-\text { traces }\right),
$$

thus forming into traceless, symmetrical rank- $n$ tensors. Substituting (4) and (2) into (1) we come to schematic expression

$$
\mathcal{M}_{\phi p}=\sum_{n=2,4, \ldots}^{\infty} C_{n} A_{n} \frac{K_{\mu_{1}} \ldots K_{\mu_{n}}}{M_{\Phi}^{n}} \Pi^{\mu_{1} \ldots \mu_{n}}
$$

where for convenience we used the notation $\Pi^{\mu_{1} \ldots \mu_{n}}=\left(p^{\mu_{1}} \ldots p^{\mu_{n}}-\right.$ traces $)$.

At this point, we comment briefly on the applicability of the analysis to actual physical states. For sufficiently heavy constituent quarks, the state $\Phi$ is localized at characteristic distances $r$ which are sufficiently small compared to non-perturbative hadronic scale $\Lambda_{Q C D}^{-1}$ to treat the system perturbatively. On the other hand, it satisfies $r \gg m_{Q}^{-1}$, so that one can 
describe the internal dynamics nonrelativistically. Although the estimates [2] show that these requirements are reliably satisfied only for quark masses exceeding $25 \mathrm{GeV}$, one finds that a Coulomb-like potential approach provides a satisfactory description of quarkonium spectroscopy (see, e.g. [9]). Hence we may also expect a one-gluon exchange picture to be relevant for $\mathrm{J} / \psi$ and $\Upsilon$ internal dynamics. We thus consider $\Phi$ as $\mathrm{SU}(3)$-Coulombic bound state characterized by a "Bohr" radius $a_{0}$ and the corresponding "Rydberg" energy $\epsilon_{0}$ :

$$
a_{0}=\frac{4}{3 m_{Q} \alpha_{s}}, \quad \epsilon_{0}=\left(\frac{3 \alpha_{s}}{4}\right)^{2} m_{Q}
$$

where $\alpha_{s}$ is the gauge coupling constant, evaluated at a scale $\epsilon_{0}$. Then the direct calculations of [2] in $S U(N)$ gauge theory give

$$
C_{n}^{(1 S)}=a_{0}^{3} \epsilon_{0}^{2-n} d_{n}^{(1 S)}, \quad d_{n}^{(1 S)}=\left(\frac{32}{N}\right)^{2} \sqrt{\pi} \frac{\Gamma\left(n+\frac{5}{2}\right)}{\Gamma(n+5)} .
$$

for the Wilson coefficients $C_{n}$ of $1 S$-quarkonium state in the leading order in $1 / N^{2}$ (analogous expressions for the $P$-states can be found in Ref. [10]).

We now insert the coefficients (7) into Eq. (5). If we neglect the trace terms in Eq. (4), or, equivalently, the corrections of order of $m_{N}^{2} / \epsilon_{0}^{2}$, we recover the sum rules used in [1] as the basis for calculating $\sigma_{N \Phi}(s)$,

$$
\int_{0}^{1} d y y^{n-2} \sqrt{1-y^{2}} \sigma_{N \Phi}\left(m_{N} / y\right)=I(n) \int_{0}^{1} d x x^{n-2} g\left(x, Q^{2}=\epsilon_{0}^{2}\right),
$$

with $I(n)$ given by

$$
I(n)=2 \pi^{3 / 2}\left(\frac{16}{3}\right)^{2} \frac{\Gamma\left(n+\frac{5}{2}\right)}{\Gamma(n+5)}\left(\frac{4}{3 \alpha_{s}}\right) \frac{1}{m_{Q}^{2}}
$$

One obvious problem in applying these sum rules to $\mathrm{J} / \psi$ interactions is that ratio $m_{N}^{2} / \epsilon_{0}^{2} \simeq$ 2.1 is not actually small and hence cannot be ignored. In other words, the trace terms entering the definition of twist-two operators (4) in the Wilson's ordering scheme must be included explicitly producing the corresponding changes in dispersion sum rules (8).

The tracelessness of the tensor $\Pi^{\mu_{1} \ldots \mu_{n}}$ () means that

$$
g_{\mu_{i} \mu_{j}} \Pi^{\mu_{1} \ldots \mu_{i} \ldots \mu_{j} \ldots \mu_{n}}=0 .
$$

The most general structure of $\Pi^{\mu_{1} \ldots \mu_{n}}$ is well known [1]

$$
\Pi^{\mu_{1} \ldots \mu_{n}}=\sum_{j=0}^{n / 2}(-1)^{j} \frac{(n-j) !}{2^{j} n !} m_{N}^{2 j} \sum_{\text {all permut. }} \overbrace{g \ldots g}^{j} \overbrace{p \ldots p}^{n-2 j}
$$

where the second sum runs over the $n ! /(n-2 j) !(2 j)$ ! terms of all possible permutations. Introducing $\lambda=\left(p K / M_{\Phi}\right)$ we can rewrite (5) as

$$
\mathcal{M}_{\phi p}=\sum_{n=2,4, \ldots}^{\infty} d_{n} a_{0}^{3} \epsilon_{0}^{2} A_{n} \sum_{j=0}^{n / 2}(-1)^{j} \frac{(n-j) !}{4^{j}(n-2 j) ! j !}\left(\frac{m_{N}^{2}}{\epsilon_{0}^{2}}\right)^{j}\left(\frac{\lambda}{\epsilon_{0}}\right)^{n-2 j}
$$


Changing the summation index $n \rightarrow n-2 j$ and making simple rearrangements of the coefficients, one obtains

$$
\mathcal{M}_{\phi p}=a_{0}^{3} \epsilon_{0}^{2} \sum_{n=0,2, \ldots}^{\infty}\left(\frac{\lambda}{\epsilon_{0}}\right)^{n} \sum_{j=0}^{\infty}(-1)^{j} d_{n+2 j} A_{n+2 j} \frac{(n+j) !}{4^{j} n ! j !}\left(\frac{m_{N}^{2}}{\epsilon_{0}^{2}}\right)^{j} .
$$

We now take the explicit expressions $(7)$ for $d_{n}$ (the term proportional to $d_{0}$ corresponds to disconnected diagrams and does not enter the amplitude of quarkonium interactions in the OPE formulation) and use the definition of $A_{n}$ as Mellin transforms [12] of the gluon distribution in a proton, evaluated at the scale $Q^{2}=\epsilon_{0}^{2}$,

$$
A_{n}=\int_{0}^{1} d x x^{n-2} g\left(x, Q^{2}=\epsilon_{0}^{2}\right)
$$

This leads to the final expression

$$
\begin{gathered}
\mathcal{M}_{\phi p}=4 \sqrt{\pi} a_{0}^{3} \epsilon_{0}^{2}\left(\frac{16}{3}\right)^{2}\left[\int_{0}^{1} d x \sum_{n=2,4, \ldots}^{\infty} x^{n-2}\left(\frac{\lambda}{\epsilon_{0}}\right)^{n} g\left(x, \epsilon_{0}^{2}\right) \times\right. \\
\times \frac{\Gamma\left(n+\frac{5}{2}\right)}{\Gamma(n+5)}{ }_{3} F_{2}\left(\frac{5}{4}+\frac{n}{2}, \frac{7}{4}+\frac{n}{2}, 1+n ; \frac{(5+n)}{2}, 3+\frac{n}{2} ;-\frac{m_{N}^{2}}{4 \epsilon_{0}^{2}} x^{2}\right) \\
\left.-\frac{m_{N}^{2}}{4 \epsilon_{0}^{2}} \frac{\Gamma\left(\frac{9}{2}\right)}{\Gamma(7)} \int_{0}^{1} d x g\left(x, \epsilon_{0}^{2}\right)_{3} F_{2}\left(1, \frac{9}{4}, \frac{11}{4} ; \frac{7}{2}, 4 ;-\frac{m_{N}^{2}}{4 \epsilon_{0}^{2}} x^{2}\right)\right] .
\end{gathered}
$$

Introducing the variable $y=m_{N} / \lambda$, we get

$$
\begin{gathered}
\int_{0}^{1} d y y^{n-2}\left(1-y^{2}\right)^{1 / 2} \sigma_{N \Phi}\left(m_{N} / y\right)= \\
I(n) \int_{0}^{1} d x x^{n-2} g\left(x, \epsilon_{0}^{2}\right)_{3} F_{2}\left(\frac{5}{4}+\frac{n}{2}, \frac{7}{4}+\frac{n}{2}, 1+n ; \frac{(5+n)}{2}, 3+\frac{n}{2} ;-\frac{m_{N}^{2}}{4 \epsilon_{0}^{2}} x^{2}\right) .
\end{gathered}
$$

Comparing Eqs. (8) and (16), we see that the inclusion of finite mass corrections effectively modifies the gluon distribution function - in the r.h.s. one should integrate including confluent hypergeometric function ${ }_{3} F_{2}(\ldots)$ as a weight. In Eq. (16), $n=2,3, \ldots$, so that in order to determine the cross section, it appears that we have to solve an infinite set of equations. However, usually the application of the sum rules to deep-inelastic leptonhadron scattering [13] and heavy meson photoproduction [5] had been limited to the values of $n \leq 5-6$ [1-5]: it was expected that perturbative calculation of the coefficient functions breaks down for large $n$. The use of Eq.(16) can effectively extend the range of applicability of the sum rule approach, and the problem of relevant $n$-interval should be re-examined. Indeed, the additional weight function ${ }_{3} F_{2}$ in the r.h.s. of (16) decreases for $x \rightarrow 1$ faster for larger $n$, making the sum rules more sensitive to the behaviour of parton distribution function (PDF) at lower values of $x$. This makes the reliability region for gluonic distribution function moments effectively wider. In what follows we study the sum rules for values $n \leq 10$.

It is evident from Eq. (16) that the behaviour of the cross section at high energy is particularly sensitive to the small $x$ region, while the threshold behaviour probes the large 
$x$ region. New data from deep inelastic scattering have led to different PDF parametrizations; hence one has to check how well the sum rules, including target mass corrections as well, are satisfied for different PDF choices. In [1], the simple schematic form

$$
g(x)=\text { const. }(k+1)(1-x)^{4}
$$

was used. Here, we have considered in addition the MRS $D_{-}^{\prime}$ and the new MRS $H$ 14 parametrizations; the latter takes into account the small- $x$ behavior observed at HERA [15]. In all cases, the left hand and right hand sides of Eq. (16) agree for $2 \leq n \leq 10$ within better than $1 \%$. The sum rules are thus well satisfied for all three PDF forms used.

Next we want to check what effect the finite mass corrections have. In Fig. 1, we therefore show the ratio of the cross section including the target mass corrections (i.e. the solution of Eq.(16)) to the same calculation without them (from Eq.(8)). It is seen that the inclusion of target mass corrections changes the resulting cross section above the threshold $(\sqrt{s}>5 \mathrm{GeV})$ by less than a factor three.

Finally we want to see if and how the cross sections obtained as solution of Eq. (16) vary for different PDF forms; we have therefore calculated $\sigma_{N \Phi}(s)$ also for MRS $D_{-}^{\prime}$ and MRS $H$. The resulting cross sections, divided by the corresponding form obtained using PDF (17), are shown in Fig. 2; here all cross sections include target mass corrections. The increase of the two MRS forms at high energy, relative to that using Eq. (17), is due to the small $x$ increase in the more realistic PDF's.

In summary, we would like to stress that including finite target mass corrections justifies the use of OPE close to the threshold and meanwhile does not change significantly the behaviour of quarkonium-hadron cross section at high energies. Besides, target mass corrections are important in providing the basis for future investigations of the role of higher terms of the multipole expansion and for a description of the entire $x$ region by the sum-rule method.

\section{Acknowledgements:}

This work was supported by the GSI under grant BISAT (D. Kh.) and by INTAS under grant 3941 (A. S. and G. Z.).

\section{References}

[1] D. Kharzeev and H. Satz, Phys. Lett. B334 (1994) 155.

[2] M. E. Peskin, Nucl. Phys. B156 (1979) 365.

[3] G. Bhanot and M. E. Peskin, Nucl. Phys. B156 (1979) 391.

[4] M. A. Shifman, A. I. Vainshtein and V. I. Zakharov, Phys. Lett. B65 (1976) 255.

[5] V. A. Novikov, M. A. Shifman, A. I. Vainshtein and V. I. Zakharov, Nucl. Phys. B136 (1978) 125.

[6] A. Kaidalov, in QCD and High Energy Hadronic Interactions, J. Trân Thanh Vân (Ed.), Edition Frontieres, Gif-sur-Yvette, 1993. 
[7] K. Gottfried, Phys. Rev. Lett. 40 (1978) 598.

[8] M. B. Voloshin, Nucl. Phys. B154 (1979) 365.

[9] D.J. Gross and F.Wilczek, Phys. Rev. 30, (1973) 1343; Phys. Rev. D8, 3633 (1973); ibid D9, (1974) 980; H.D.Politzer, Phys. Rev. Lett. 30, (1974) 1346; Phys. Rep. 14C, (1974) 129.

[10] D. Kharzeev, CERN-TH/95-342, nucl-th/9601029.

[11] H. Georgi and H.D. Politzer, Phys. Rev. D14, (1976) 1829.

[12] G. Parisi, Phys. Lett. B43 (1973) 207; B50 (1974) 367.

[13] A. De Rujula, H. Georgi, and H. D. Politzer, Ann. Phys. 103 (1977) 315.

[14] A. D. Martin, R. G. Roberts and W. J. Stirling, Int. J. Mod. Phys. A10, (1995) 2885.

[15] ZEUS Collaboration, Phys. Lett. B316, 412 (1993); H1 Collaboration, Nucl. Phys. B407, 515 (1993).

\section{Figure Captions:}

Fig. 1: The ratio of $\sigma_{J / \Psi-N}(s)$, including target mass corrections, to the corresponding form without these corrections; both forms are calculated with PDF (17).

Fig. 2: The ratio of $\sigma_{J / \Psi-N}(s)$, from MRS D-' PDF (solid line) and from MRS H (dashed line), to the cross section obtained from PDF (17); all cross sections include target mass corrections. 

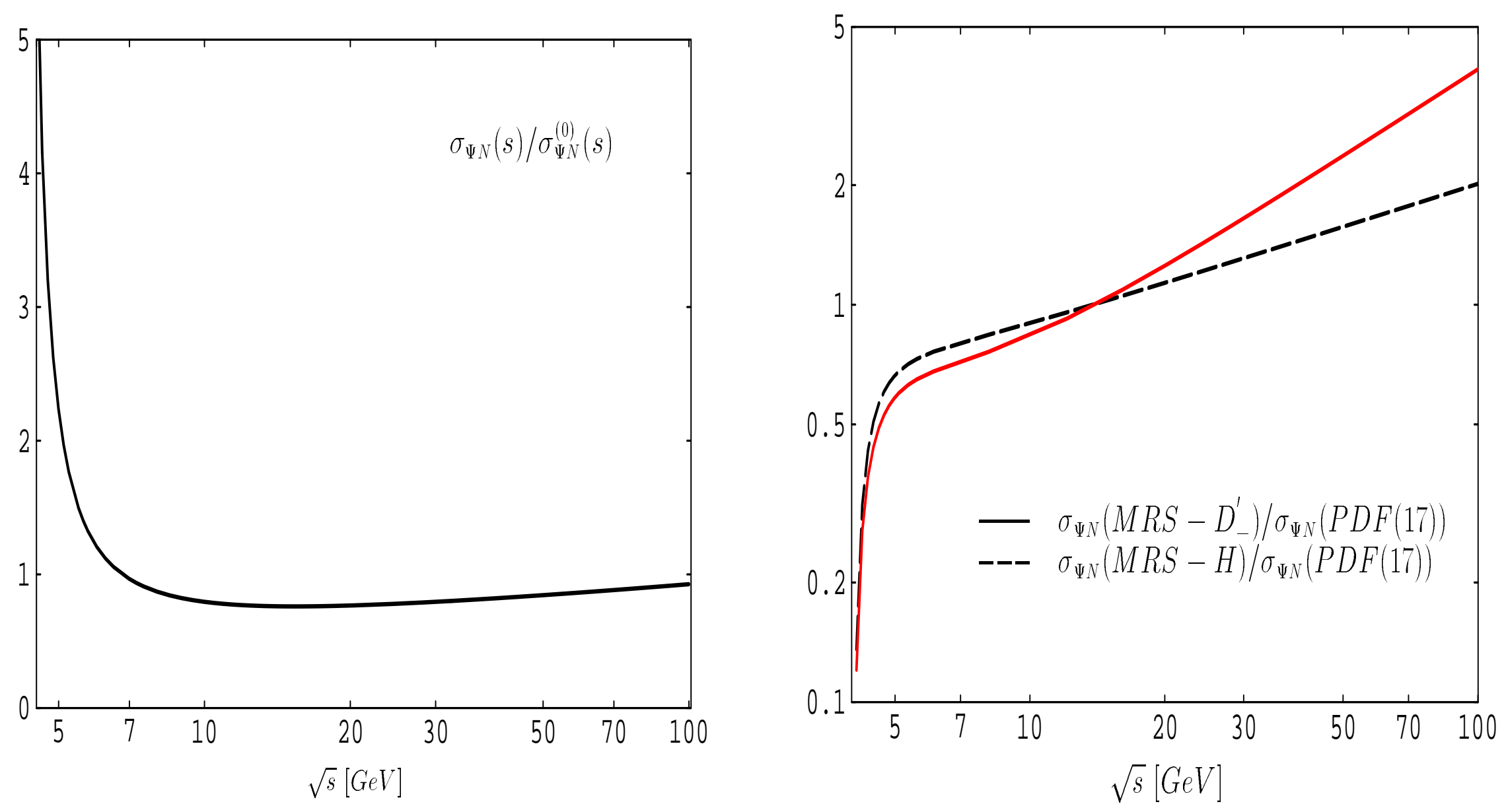

Fig. 1

Fig. 2 